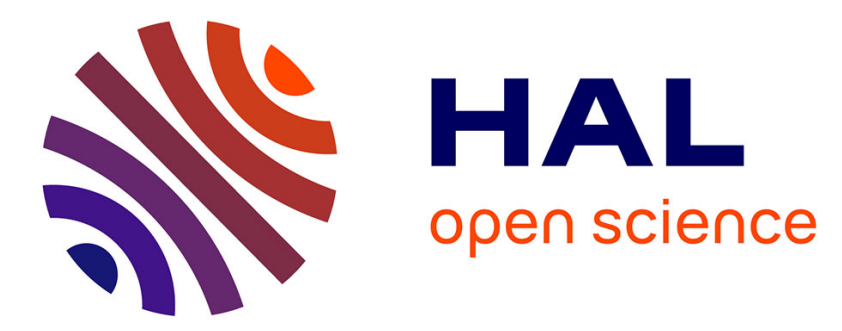

\title{
Solitary waves in discrete media with anomalous compressibility and similar to "sonic vacuum" \\ V. Nesterenko
}

\section{To cite this version:}

V. Nesterenko. Solitary waves in discrete media with anomalous compressibility and similar to "sonic vacuum". Journal de Physique IV Proceedings, 1994, 04 (C8), pp.C8-729-C8-734. 10.1051/jp4:19948112 . jpa-00253354

\section{HAL Id: jpa-00253354 https://hal.science/jpa-00253354}

Submitted on 1 Jan 1994

HAL is a multi-disciplinary open access archive for the deposit and dissemination of scientific research documents, whether they are published or not. The documents may come from teaching and research institutions in France or abroad, or from public or private research centers.
L'archive ouverte pluridisciplinaire HAL, est destinée au dépôt et à la diffusion de documents scientifiques de niveau recherche, publiés ou non, émanant des établissements d'enseignement et de recherche français ou étrangers, des laboratoires publics ou privés. 


\title{
Solitary waves in discrete media with anomalous compressibility and similar to "sonic vacuum"
}

\author{
V.F. Nesterenko
}

Lavrentyev Institute of Hydrodynamics, Lavrentyev Avenue 15, Novosibirsk 630090, Russia

\begin{abstract}
Propagation of deformation pulses in significantly nonlinear media with inner structure where there is no linear part in interaction force between neighboring structure elements is considered. This makes impossible the application of traditional wave equation or developed earlier approaches wherein nonlinearity serves as a small correction and requires the development of a qualitatively new nonlinear wave dynamics.
\end{abstract}

Résumé. La propagation des impulsions de déformation dans un milieu non linéaire est considérée. La structure interne est telle qui'il n'existe aucune partie linćaire dans les forces d'interaction entre les structures voisines. Cette hypothèse rend impossible l'application de l'equation d'onde traditionnelle mais aussi les approches développées précédemment pour lesquelles la non linéarité est une correction mineure.

Aussi, nous proposons le développement d'une nouvelle formulation essentiellement non linéaire de la dynamique des ondes.

\section{SONIC VACUUM}

Theoretical consideration. The propagation of compression pulses in the materials where longwave sound velocity $c_{0}$ is equal to zero or small as compared to the phase velocity of disturbances is considered in [1-10]. For such media the concept of "sonic vacuum" is introduced implying the impossibility for linear waves described by standard wave equation to propagate in them, or their insignificance for description of strongly nonlinear disturbances. The example of such elastic nonlinear media in one-dimensional case are the systems with the dependence of stress on the deformation of type $\sigma=b \xi^{n}, n>1$. In the case $n=3 / 2$ corresponding to the Hertz law for the contact of spheres the solitary waves of new type are found in numerical calculations, analytically within the long-wave approximation and experimentally $[1-4,6,8]$. For arbitrary $n>1$ the existence of similar stationary solutions is found for long-wave equation $[5-7]$ :

$$
u_{t t}=-c_{n}^{2}\left\{\left(-u_{x}\right)^{n}+\frac{n a^{2}}{24}\left[(n-1)\left(-u_{x}\right)^{n-2} u_{x x}^{2}-2\left(-u_{x}\right)^{n-1} u_{x x x}\right]\right\}_{x}, \xi=-u_{x}>0,
$$

where $u$ is the displacement, $a$ is the initial distance between the particles of undisturbed system, $c_{n}$ is the constant with the velocity dimensionality. The requirement $\xi>0$ is necessary for the case of discrete systems with zero tensile strength and for certain values of $n$.

In [7] equation (1) is written conveniently for specific applications.

For the reason cited in [11] the substitution of term with higher derivatives with respect to $x$ for the term with mixed derivatives with respect to $x, t$ is considered more preferable for wave equations with spatial dispersion. This preserves the equivalency of the equations in the considered long wave limit, but in linear case eliminates imaginary frequencies in dispersion relation when $\lambda<\lambda_{\min }=\pi a / \sqrt{3}$, although in this case neither one, nor another equation may be used correctly. Substituting the lower approximation for the term with the higher derivative in square brackets, obtain the more "preferable" equation:

$$
u_{t t}=-\left\{c_{n}^{2}\left(-u_{x}\right)^{n}-\frac{a^{2}}{12}\left[u_{t t x}+\frac{c_{n}^{2} n(n-1)}{2}\left(-u_{x}\right)^{n-2} u_{x x}^{2}\right]\right\}_{x} .
$$


The equations $(1,2)$ may be obtained [7] also using the variational approach with appropriate Lagrangians in designations [11]:

$$
\begin{gathered}
L_{1}=\frac{u_{t}^{2}}{2}-c_{n}^{2}\left\{\frac{\left(-u_{x}\right)^{n+1}}{n+1}+\frac{a^{2}}{2}\left[\frac{n}{4}\left(-u_{x}\right)^{n-1} u_{x x}^{2}-\frac{1}{3}\left(-u_{x}\right)^{n} u_{x x x}\right]\right\} \\
L_{2}=\frac{u_{t}^{2}}{2}+\frac{a^{2}}{24} u_{x t}^{2}-c_{n}^{2}\left[\frac{\left(-u_{x}\right)^{n+1}}{n+1}-\frac{\left(-u_{x}\right)^{n}}{24} u_{x x x}\right]
\end{gathered}
$$

Lagrangian formalism was used in [10] to prove the stability of periodical solutions (1) in the case $n=3 / 2$.

Write down also the equation (2) in deformations $\xi$

$$
\xi_{t t}=\left\{c_{n}^{2} \xi^{n}+\beta \xi_{t t}-\gamma \xi^{n-2} \xi_{x}^{2}\right\}_{x x}, \beta=\frac{a^{2}}{12}, \gamma=\frac{a^{2} c_{n}^{2}}{24} n(n-1) .
$$

It should be noted that $c_{n}$ is not a long-wave sound speed $c_{0}$, the expression for which, as it may be easily seen from (5), has the form

$$
c_{0}=c_{n} \sqrt{n} \xi_{0}^{\frac{n-1}{2}}
$$

tending to zero in the case when $\xi_{0} \rightarrow 0$ at $n>1$.

Stationary solutions $\xi(x-V t)$ of eq. (1) may be obtained. In this case upon integration with respect to $x$ and the change of the variables $(\xi, x) \rightarrow(y, \eta)$ eq.(1) reduces to the equation of motion of nonlinear oscillator in potential field $W(y)$

$$
\begin{gathered}
y_{\eta \eta}=-\frac{\partial W(y)}{\partial y}, W(y)=\frac{y^{2}}{2}-\frac{n+1}{4} y^{\frac{4}{n+1}}+C y^{\frac{2}{n+1}}, \\
\eta=\frac{x}{a} \sqrt{\frac{6(n+1)}{n}}, y=\xi^{\frac{n+1}{2}}\left(\frac{c_{n}}{V}\right)^{\frac{n+1}{n-1}}
\end{gathered}
$$

where $C$ is the constant. In particular, there is a partial periodical solution of $(1,7)$

$$
\xi=\left\{\frac{(n+1)}{2} \frac{V^{2}}{c_{n}^{2}}\right\}^{\frac{1}{n-1}}\left|\sin \left[\frac{n-1}{n+1} \sqrt{\frac{6(n+1)}{n}} \frac{(x-V t)}{a}\right]\right|^{\frac{2}{n-1}}
$$

The solution ( 8 ) has the form of a sequence of jointed in $\xi_{0}=0$ (to say more precisely in $\xi_{0} \rightarrow 0$ [7]) of positive humps. For $n=3 / 2$ (a chain of adjacent elastic spheres) we have $[1,8]$

$$
\xi=\left(\frac{5}{4} \frac{V^{2}}{c_{3 / 2}^{2}}\right)^{2} \cos ^{4} \frac{\sqrt{10}}{5 a}(x-V t) .
$$

Modulation stability of the periodical solution (9) is proved in [10]. At $n=3$, corresponding to transverse vibrations of linearly elastic unstressed fiber with discrete masses [9], or linear gas-liquid system, gas being near the critical point [12], there is a partial periodical solution (no restriction of $\xi>0$ type is required)

$$
\xi=\sqrt{2} \frac{V}{c_{3}} \sin \frac{\sqrt{2}}{a}(x-V t)
$$

The solutions $(1,7)$ as a solitary wave exist when the integration constant $C$ is within the limits

$$
0<C<\frac{n^{2}-1}{2} n^{\frac{n}{1-n}}
$$

Physical sense of these restrictions is that finite, though as small as one likes initial deformation $\xi_{0}$ (sound velocity $c_{0}$ ) is necessary and solitary compression wave is of supersonic $\left(V>c_{0}\right)$ nature. At

$$
C \nprec \frac{n^{2}-1}{2} n^{\frac{n}{1-n}}
$$


these solitary waves transform into the $\mathrm{KdV}$ equation solitons. If $C$ is close to zero, which corresponds to $\xi_{\max } \gg \xi_{0}$, the solitary waves qualitatively differ from the $\mathrm{KdV}$ solitons. In this case they are the main carrying tons of these strongly nonlinear system (called "nestons" [5-7]) characterized by the following dependence of phase velocity $V$ on $\xi_{\max }$, or on maximum particle velocity $v_{\max }$ and characteristic spatial length $L_{n}$ :

$$
V=c_{n} \sqrt{\frac{2}{n+1}}\left(\xi_{\max }\right)^{\frac{n-1}{2}}=\left(\frac{2 c_{n}^{2}}{n+1}\right)^{\frac{1}{n+1}} \cdot v_{\max }^{\frac{n-1}{n+1}}, L_{n}=\frac{\pi a}{n-1} \sqrt{\frac{n(n+1)}{6}} .
$$

Note, that the phase velocity and the width of "nestons" are independent of the sound velocity $c_{0}$, as opposed to the KdV equation solitons, inheriting the medium properties in linear description.

One may expect that analogous significantly nonlinear periodical and solitary waves (at $\xi_{\max } \gg$ $\xi_{0}$ ) will be typical of all discrete systems of the type of "sonic vacuum", i.e. where the law of interaction between the neighboring structure elements will provide the equality to zero of long-wave sound velocity at $\xi_{0}=0$, but not only for power dependence [13].

Numerical calculations of discrete system of particles in the case $n=3 / 2[1,8]$ have shown that discovered solitary waves for long-wave approximation $(1,7)$ practically coincide with solitary waves of discrete system, while the latter conduct at the interaction analogously to solitons. Note that the peculiarities of the pulses in the system with chaotic particle sizes are studied in $[1,8]$.

Finally note that in "sonic vacuum" (assuming that the soliton is well described at $\xi_{0}=0$ by one hump of periodical solution (8)) the following connection between the impulse and total energy of the given quasi-particle may be obtained:

$$
E_{0}=\frac{p^{2}}{2 m_{e f}}, m_{e f}=\alpha m
$$

where $m$ is the mass of the particles of the discrete system, $\alpha$ - coefficient depending on $n$.

Experiment. Experimental studies of the disturbances in the "sonic vacuum" including the system with two masses are reported in $[2,3,4,6,8,14]$ by the example of a one-dimensional chain of the particles. It is worth noting that the solitary waves in two-mass system of spherical particles $(n=3 / 2)$ have spatial size equal to five sizes of elementary cell composed of light and heavy particles. Different compression pulses registered by piezoelectric transducers located either near the contact of the particle system with rigid wall or inserted in the middle of a cut granule are presented in Fig. 1. Experimental data are in qualitative agreement with the results of numerical calculations $[2,3,4,8]$. A discrepancy is caused by the fact that experimentally found dissipation is disregarded in the calculations. If the dissipation is qualitatevely significant, as in the system of lead granules [3], then in spite of the system discreteness the compression pulse at the same loading regimes is of monotonous character (Fig. $1 \mathrm{c}$ ), as opposed to the system of elastic steel granules (Fig. $1 \mathrm{~b}$ ).

Of peculiar interest is the behavior of compression pulses at the contact of two "sonic vacuums". Actually, there is no concept of sonic impedance determining the reflected and passed impulse amplitudes. The behavior of compression pulses when passing through the contact of two "sonic vacuums" - the chains of steel spherical granules with diameters $4.75 \mathrm{~mm}$ (SV-1) and $7.9 \mathrm{~mm}$ (SV-2) was studied in the experiments (Fig. $1 \mathrm{~d}, \mathrm{e}$ ) and numerical calculations [14]. The compression stress was measured both in incident and reflected waves (transducer in the 15th particles from the contact) and in passed impulses (transducer in the 6th particle from the contact).

The following results are obtained. If the soliton falls at the contact from the side of SV-2 ("heavy") (Fig. 1 d), then the reflected compression pulse in it is not registered, while in SV-1 a significantly asymmetrical passed triangular pulse with steep front arises near the contact, which later decomposes as it propagates inside the system over a series of 6 solitons (in numerical calculations). In the experiments the sequence of solitons reflected from the rigid wall on which the complete chain rests (Fig. $1 \mathrm{~d}$ ) is registered due to the shortness of the system (20 "light" and 20 "heavy" particles on the whole). When the soliton runs against the contact from the side of SV-1 ("light") a reflected soliton appears in it and the soliton in SV-2 (Fig. $1 \mathrm{e}$ ). It is apparent that such a pattern of pulse interaction with the interface of two "sonic vacuums" qualitatively differs from that observed in traditional acoustics.

The impulse parameters for the case corresponding to Fig. 1 e may be found from the laws of conservation. Actually, the total impulse and energy should be preserved in the system

$$
p_{0}=p_{1}-p_{2}, \quad E_{0}=E_{1}+E_{2},
$$



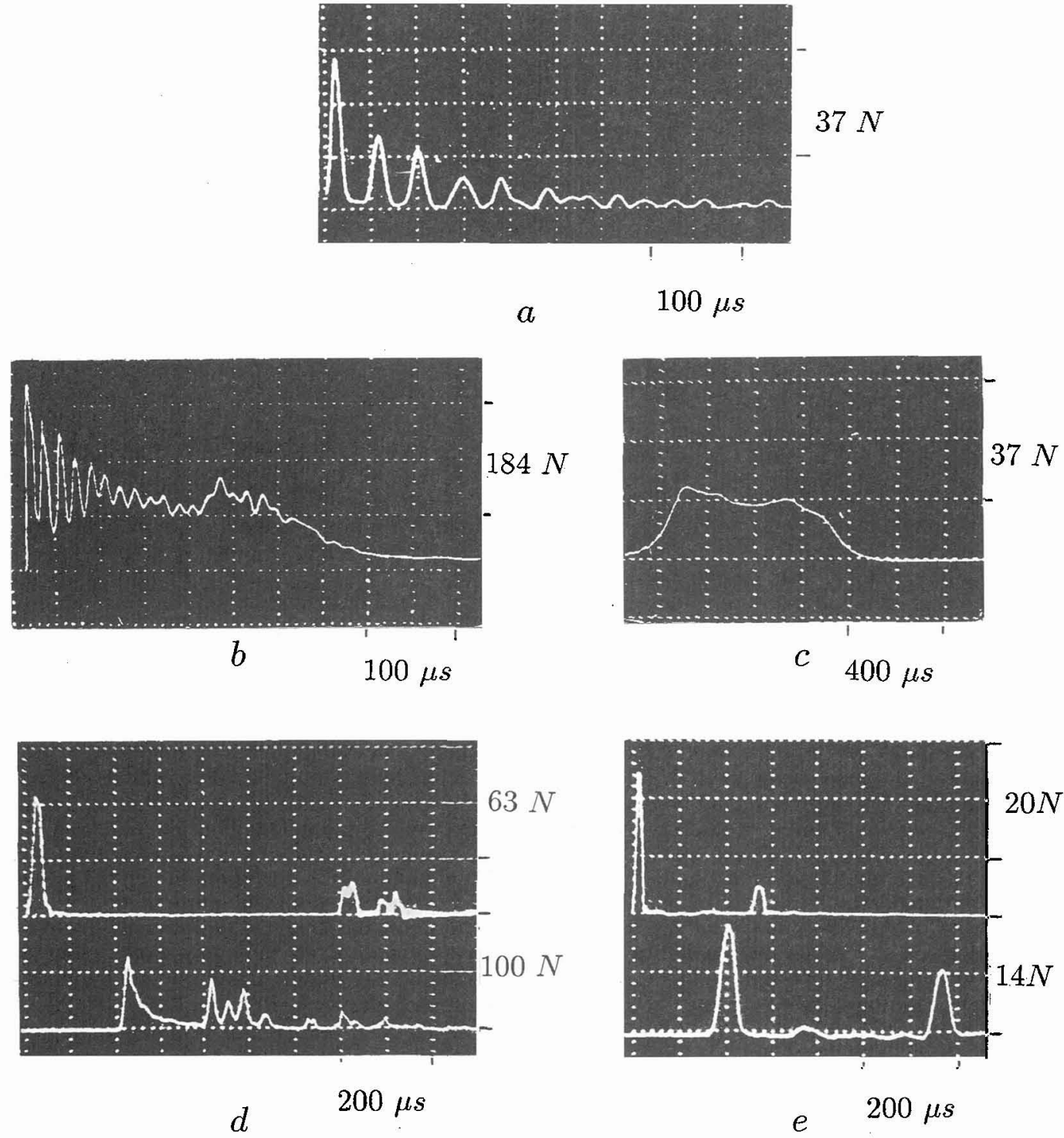

Fig. 1. $a-$ compression force on the contact of one-dimensional chain of steel spheres with rigid wall at piston impact (sphere diameter $d=4.75 \cdot 10^{-3} \mathrm{~m}$, their number $N_{s}=40$, piston mass $M=5 \mathrm{~m}$, velocity $v_{p}=0.5 \mathrm{~m} / \mathrm{sec}$ ), $b$ - the same at $N_{s}=20, M=30 \mathrm{~m}, v_{p}=1 \mathrm{~m} / \mathrm{sec}$;

$c$ - the same for the cnain of lead spheres at the same geometry and loading conditions as in $b$;

$d$ - compression force in spheres after piston $\left(M=m_{2}, v_{p}=1 \mathrm{~m} / \mathrm{sec}\right)$ impact before and behind contact SV-2 $\div$ SV-1 in incident wave (upper trace, 1-st pulse), passed (lower trace, 1-st pulse), and in the wave reflected from rigid wall;

$e$ - the same after piston $\left(M=m_{1}, v_{p}=1 \mathrm{~m} / \mathrm{sec}\right)$ impact before and behind contact SV-1 $\div \mathrm{SV}-2$ in incident wave (upper trace, 1-st pulse), reflected (upper trace, 2-nd pulse), passed (lower trace, 1 -st pulse) and in the wave reflected from rigid wall. 


$$
E_{0}=\frac{p_{0}^{2}}{2 m_{1 c f}}, \quad E_{1}=\frac{p_{1}^{2}}{2 m_{2 e f}}, \quad E_{2}=\frac{p_{2}^{2}}{2 m_{1 e f}},
$$

where $p_{0}, p_{1}, p_{2}$ are the impulses of incident, passed and reflected solitons respectively. Hence the impulses $p_{1}, p_{2}$ of passed soliton may be obtained as follows:

$$
p_{1}=\frac{2 p_{0}}{1+k}, p_{2}=\frac{1-k}{1+k} p_{0}, \text { where } k=\frac{m_{1}}{m_{2}} .
$$

The dependences between $p_{0}$ and $p_{1}, p_{2}$ qualitatively conform with the results of measuring the appropriate forces (Fig. $1 \mathrm{~d}$, e), and in good agreement with computer calculations [14]. It is apparent that such a pattern is possible only when the pulse falls from "light" SV into "heavy" SV. At the same time the laws of conservation ensure realization of only one of decomposition mechanisms, whose uniqueness calls for justification.

The transformation of the pulses at the contact (Fig. $1 \mathrm{~d}$, e), the rapid decomposition of initial disturbance with steep front into the sequence of pulses (Fig. $1 \mathrm{a}, \mathrm{b}$ ) allow the systems of "sonic vacuum" type and their contacts to efficiently transform external actions on the system in the pulse sequence required.

\section{MEDIUM WITH ANOMALOUS COMPRESSIBILITY}

The existence of stationary solutions in discrete medium with power interaction law but at $0<n<1[13]$ is of interest. The given value $n$ stipulates anomalous behavior under compression, i.e. the decrease of its elasticity modulus as the deformation grows. This is typical of the media subjected to phase transition or failure. Appropriate long-wave approximation is analogous to (1), and as well as in the case $n>1$ for stationary solutions it may be reduced to the equation of nonlinear oscillator in potential field of type (7).

At

$$
0>C>N_{1}=\frac{n^{2}-1}{2} n^{\frac{n}{1-n}}
$$

$W(y)$ will have two extremes $y_{1}, y_{2}$, with $y_{1}>y_{2}\left(y_{1}, y_{2}\right.$ correspond to the minimum and maximum respectively). Note that relative arrangement of extremes when $0<n<1$ is reverse to their location for $n>1$ at corresponding values of the constant $C$. If

$$
\frac{n-1}{2}\left(\frac{n+1}{2 n}\right)^{\frac{n}{n-1}}=N_{2}>C>N_{1}
$$

then the curve $W(y)$ will be placed at right lower quadrant of the plane $W(y) \div y$. At $C=N_{2}$ the curve $W(y)$ will touch the abscissa axis $y$ in the maximum point $y_{20}=(2 n /(n+1))^{\frac{n+1}{2(1-n)}}$, while at $C \rightarrow N_{1}$ both extremes will combine in one point $y_{0}=n^{\frac{n+1}{2(1-n)}}$.

According to its physical sense $y_{2}$ corresponds to the initial state of the system. The relative arrangement of $y_{1}$ and $y_{2}$ permits a conclusion on the possibility of existence of periodical waves and solitary rarefaction waves at $\xi>0$ in the case (16). Solitary compression waves in the system are absent in contrast to the values $n>1$. If physical sense admits negative values of $\xi$, then solitary waves are possible at $0>C>N_{2}$.

Using the mentioned properties of the function $W(y)$, one may derive the dependence of phase velocity of solitary rarefaction wave on deformation in its minimum $\left(\xi_{\min } \ll \xi_{0}\right)$ at total "energy" of nonlinear oscillator $W_{0}=W\left(y_{2}\right)$ and providing the (16)

$$
V=c_{n}\left\{\frac{\left(n^{2}-1\right) \xi_{0}^{2}}{4\left[\xi_{0} n-\xi_{\min }(n+1)\right] C}\right\}^{\frac{n-1}{2}} .
$$

If $\xi_{\min } \rightarrow 0$, which corresponds to $C \rightarrow N_{2}$ from (17) it is easily seen that

$$
V \rightarrow c_{n} \xi_{0}^{n-1 / 2} \sqrt{\frac{2 n}{n+1}} .
$$

At $C \rightarrow N_{1}$

$$
V \rightarrow c_{n} \sqrt{n} \xi_{0}^{n-1 / 2}
$$


Since at the initial state the long-wave sound velocity $c_{0}=c_{n} \sqrt{n} \xi_{0}^{n-1 / 2}$, then a conclusion may be made on supersonic character of solitary rarefaction wave in the system with $0<n<1$ in reference to the initial state. This property is easily seen from the relation

$$
\frac{V^{2}}{c_{0}^{2}}=\frac{1}{n} y_{2}^{\frac{2(1-n)}{n+1}}
$$

and limiting values of $y_{2}$ conforming to the condition (16).

Characteristic spatial size of solitary rarefaction wave is, for example, at $n=1 / 2, L_{1 / 2} \simeq 20 a$.

\section{CONCLUSIONS}

It is appropriate to note that in the vicinity $n=1$ for the systems with power interaction law a peculiar "sonic catastrophe" is observed. Actually, whatever small was the deviation of $n$ from 1 as compared to 1 the stationary solitary waves of rarefaction (at $n>1$ ) or compression (at $n<1$ ) become prohibited. Besides, if for $n<1$ the velocity of solitary rarefaction waves at $\xi_{\min } / \xi_{0} \ll 1$ is determined by $\xi_{0}$ and tends to $c_{0}$ at $n \rightarrow 1$, then for $n>1$ at $\xi_{\max } / \xi_{0} \gg 1$ the compression wave velocity depends only on $\xi_{\max }$ and does not tend to the sound velocity at $n \rightarrow 1$. A distinctive feature of such systems is a commensurability of characteristic spatial scales of essentially nonlinear disturbance to inner size of the medium structure.

The results of numerical and experimental study of passage processes of compression pulses through the interface of two "sonic vacuums" with different microstructure clearly demonstrate new qualitative peculiarities of wave dynamics in tested materials as compared with traditional linear and nonlinear acoustics. The wave properties of these systems may be applied for desirable transformation of pulsed disturbances.

\section{ACKNOWLEDGEMENTS}

The work is supported by Russian Foundation for Fundamental Researches (93-02-14880) and International Science Foundation.

The author thanks A.N. Lazaridi for aid in performing the experiments and E.B. Sibiryakov for the help in realization of numerical calculations.

\section{REFERENCES}

1. Nesterenko V.F., Prikl. Mekh. Tekh. Fiz., 5 (1983) 136-148.

2. Lazaridi A.N., Nesterenko V.F., Prikl. Mekh. Tekh. Fiz., 3 (1985) 115-118.

3. Nesterenko V.F., Lazaridi A.N., "Solitons and shock waves in one-dimensional granular media," XI International Symposium on Nonlinear Acoustics, Novosibirsk, 24-28 August 1987, Part 1, pp. 309-313.

4. Nesterenko V.F., Lazaridi A.N., "The peculiarities of wave processes in periodical systems of particles with different masses," Obrabotka materialov impulsnymi nagruzkami, Novosibirsk, 1990, pp. 30-42.

5. Nesterenko V.F., Fizika Goreniya i Vzryva, 3 (1992) 121-123.

6. Nesterenko V.F., "Pulse compression nature in strongly nonlinear medium," Second Intern. Symp. on Intense Dynamic Loading and Its Effects, Chengdu, China, 23-28 June 1992, pp. $236-240$.

7. Nesterenko V.F., "A new type of collective excitations in a "sonic vacuum", Akustika neodnorodnykh sred, Novosibirsk, 20-23 April 1992, pp. 228-233.

8. Nesterenko V.F., High-rate Deformation of Heterogeneous Materials (Nauka, Novosibirsk, 1992) Ch.2, pp. 51-80.

9. Nesterenko V.F., Fizika Goreniya i Vzryva, 2 (1993) 132-134.

10. Gavrilyuk S.L., Nesterenko V.F., Prikl. Mekh. Tekh. Fiz., 6 (1993) 45-48.

11. Whitham G.B., Linear and Nonlinear Waves (New York-Toronto, Wiley-Interscience Publ., 1974) pp. 443-445.

12. Gavrilyuk S.L., A model of plug-chain system near the thermodynamic critical point: mechanical analogy, explicit solutions, long-wave approximation, connection with the Korteweg theory of capillarity and modulation equations, Prepublication on Rapport, Ecole Normale Superieure de Lyon, No. 115, 1993.

13. Nesterenko V.F., Fizika Goreniya i Vzryva, 2 (1993) 134-136.

14. Nesterenko V.F., Lazaridi A.N., Sibiryakov E.B., Prikl. Mekh. Tekh. Fiz., 2, (1995) (in press). 